\title{
Tablet App for Child Cognitive Assessment in Low and Middle Income Countries
}

Patricia Francis-Lyon, Yasser Attiga, Rashmi Manjunath, Uma Ramasubramanian, Vaishali Chaudhuri, Tri Nguyen, Xiangyi Xu, Siyang Zeng Department of Health Informatics, University of San Francisco. Email: pafrancislyon@usfca.edu

Amina Abubakar, Charles R Newton KEMRI, Wellcome Trust. AAbubakar@kemri-wellcome.org

\begin{abstract}
Sub-Saharan Africa is home to millions of children who face challenges in achieving their cognitive potential due to chronic poverty and other factors such as malaria and HIV infection. Scarcity of resources during early developmental stages may contribute to developmental delay in various domains, including motor, language and social-emotional, that may affect quality of life into adulthood. However, early identification of developmental delay enables early intervention, often resulting in developmental gains and a lifetime of improved capacity and fulfillment.
\end{abstract}

A team from the Neuroscience research unit at Kenya Medical Research Institute (KEMRI) has been actively engaged in developing and adapting measures of child development for use in Low Income Countries. Currently, assessments are conducted with paper, pencil and stopwatch. In collaboration with a Health Informatics team from the University of San Francisco (USF), a tablet app was designed and developed to replace paper and pencil assessments of children ages 6-60 months, with the aim of improving the data collection process and the integrity of the resulting data. The app is in the prototype stage, and is expected to be field-tested and evaluated next year.

Keywords - mHealth; data collection; cognitive research; mobile app design and development

\section{INTRODUCTION}

Childhood development has become a focus of the global community in recent years, as evidence has indicated that at least 200 million children fall short of reaching their full potential due to the impact of factors associated with poverty at this crucial stage of life [1]. The impact of the first five years of physical, cognitive and social growth is especially influential [2]. The consequences of developmental delay for children last a lifetime and typically affect their progeny, continuing the cycle of poverty. Motor, cognitive, language and social-emotional development are impacted by a variety of factors such as dietary deficiencies, exposure to heavy metals, chronic infections, unstimulating home environments, maternal depression and exposure to violence [1,2]. Developmental delays can affect children's performance in school, impacting their skills and employment prospects in adulthood.

Early detection (by age 5 years) of developmental delay can identify children for early intervention therapies to address cognitive, physical and social development, resulting in improved outcomes during schooling and into adulthood. Measures of cognitive ability are needed to both identify children for intervention, and to assess the efficacy of such efforts. The development and adaption of measures to assess cognitive, motor, language and social-emotional function in early childhood have thus been undertaken by psychologists in developing nations $[3,4]$.

Recently, psychologists in developing nations have been calling for modernizing forms of data collection utilized in cognitive assessments. Tablet-based assessments are proposed to reduce time and monetary costs while maintaining data integrity [5]. Tablet-based versions of early grade assessments in math and reading have been developed for international use [6,7], and cognitive assessment apps have been developed by testing institutions to replace paper and pencil format [5]. Also, InterNDA, developed for multi-dimensional comparison of "optimally healthy" international preschool children from middle and upper class families, contains a tablet component [8].

Research has indicated that in order to be accurate, cognitive assessments must be developed and administered within the cultural context [9]. For example, in rural Kenya the construct of intelligence includes social qualities, practical thinking, comprehension, and academic achievement. Of these four dimensions only one aspect, academic achievement, correlates with Western measures [10]. Thus, there is a need for local development of cognitive assessment tools. Principles for the selection, adaptation, and evaluation of tests to assess child cognition in developing nations have been proposed [11].

Kenya is active in the development of child cognitive assessment tools. Instruments to assess cognitive functioning, including motor skills, executive function, and language abilities, were developed specifically for children of rural Kenyan communities [12]. Efforts to develop and adapt measures of child cognitive development for use in low income nations are being conducted by a team from the Neuroassessment unit at Kenya Medical Research Institute (KEMRI). In collaboration with the Health Informatics program from the University of San Francisco (USF), a prototype tablet app was designed and developed to replace paper and pencil assessments of cognitive function in preschool children, with the aim of improving the data collection process and the integrity of the resulting data. The tablet app contains the same tests as the paper assessment, developed for the local Kenyan population. This provides an opportunity for executing a case study on the impact of moving from paper to tablet-based cognitive assessment when fieldtesting begins. Our low-cost collaboration utilizing freely 
available tools produced an app that is customizable to the local setting, and our process is suitable for cross-continental collaboration.

\section{METHODS}

\section{A. Child Assessment}

In a review of child development assessment tools for use in low-income and middle-income countries, five key global developmental domains were identified as important in the assessment of a child's developmental progress: (i) cognitive skills, including memory and information processing; (ii) language skills, including receptive and expressive language; (iii) motor skills, including fine motor and gross motor skills; (iv) social and emotional skills; including the ability to understand their own and others emotional states; and (v) adaptive behavior skills, including conceptual, social, and practical skills for everyday functioning [13]. The current KEMRI paper and pencil tests, upon which our app is based, assesses these developmental domains, utilizing tests such as number recall and reproducing picture shapes.

The content in the app was developed based on measures previously developed and validated in Kilifi Kenya. The gross motor and fine motor items were borrowed from the Kilifi Developmental Inventory, a locally developed measures which has been standardized and validated for Kilifi [14]. The other sections such as the digit span and category fluency were adapted from measures previously developed in Kilifi for assessing cognitive functions among school-aged children [15]. Both the digit span and category fluency are based on wellestablished measures used in other parts of the world. The use of an app to record the child's speech, including the words and digits, will allow for later verification of the responses by the child. The subsection on the social, emotional and behavioral development is based on a locally developed measure known as the profile of social and emotional development. This section uses a conversational approach to elucidating various aspects of child's social emotional development. The current content targets to assess children across multiple developmental domains from the age of 6 months to 60 months.

\section{B. Collaborative Design of Tablet App}

The user-centered "Design Thinking" methodology of Stanford d.school [16] is taught in USF's Mobile Health App Design and Development course as part of the Health Informatics Masters program. Core concepts of this methodology that were utilized in the design of the tablet app were:

- empathize with user: interview, observe, engage, watch and listen as users demonstrates their situation

- define problem: develop system insights, identify needs of users, synthesize your findings

- ideate: defer judgement, "go wide" creating a variety of ideas that user may react to

- prototype: build quick mockups to place in user's hands to test variables. Build prototypes that can fail quickly and cheaply
- test: experiment rapidly and iteratively with user, who should feel free to criticize, discard, merge your ideas. Users are hesitant to constructively criticize: build a collaboration where criticism is welcome.

Intercontinental collaborative design was facilitated utilizing InVision [17], a state-of-the-art collaborative design tool used by Silicon Valley companies such as Twitter and Netflix, and provided freely for educational use. InVision provides rapid user interface (UI) prototyping with no coding, so researchers in Kenya could quickly see a tablet mockup of the app and test its UI. From Kenya researchers navigated through the prototype, discussing design issues with the USF informaticists located in the United States. Brainstorming, prototyping, testing and improving ideas were carried out via online video conference, enabling the team to arrive at optimal solutions.

The empathetic design process uncovered a number of "pain points" i.e. a real or perceived problem with the current system using paper, pencil and timer for child cognitive assessment:

Data collectors' pain points:

1. process is too busy: can't write what children are speaking and score correct/incorrect quickly enough (while keeping time)

2. young children become restless during the long test

3. some answers to questions conflict with each other or are of the wrong type

4. some questions are inadvertently skipped

5. some questions should not be asked, if the answer to a previous question renders them irrelevant

6. many rural homes do not have addresses, so description of location can be challenging

Data manager's pain points:

1. need for transcribing written notes to electronic format for data analysis and storage

2. data integrity: busy process can result in bad data, with no means of verification

3. difficult to decipher scribbled notes of data collectors

4. validation: some errors require reconnecting with subjects, who may be geographically distant

5. need to audit transcribed notes

To address the data collectors' pain points, it was decided to: 1) utilize tablet capabilities to replace writing down the child's speech with automatically-timed recording so that the data collector needs only score correct/incorrect using the tablet toggle switch; 2) take advantage of children's fascination with mobile technology to hold their interest, and use color to make the test more attractive; 3) constrain answers with toggles, radio buttons and date-pickers depending on type of question and employ some automatic validation for answers; 4) employ an automatic warning for unanswered questions and implement easy navigation with side-swipe menu as well as "Next" and "Back" buttons; 5) employ conditional branching where some questions are automatically skipped over if the answer to a previous question renders them irrelevant; and 6) 
employ GPS functionality of the tablet to automatically assign longitude and latitude for location information.

The data manager's pain points were addressed by the app's automatic export to csv files. This replaced deciphering and transcribing hand-written notes, realizing decreased costs for data entry. Worker-entered data is automatically saved, along with audio and image files of primary data, in a timestamped folder labeled with the child's study ID. The audio recording can be accessed to verify that scoring (no longer a busy process) was accurate. The need for data integrity is addressed with automatic saving of a revision history for each individual test, as a backup for the master file that contains in final form the data from all the child's tests. Data can now be dragged and dropped rather than transcribed, obviating the need for most of the auditing. Data files are now doubleclicked to open in Excel for easy data analysis.

The tablet app was designed to initially employ the same tests as the paper assessment it is replacing. However, it was decided to create an app that is easily modifiable by researchers, who are able to add, delete or modify the content of a test question by rewriting the plain text file from which the app pulls content to render the screen.

\section{Coding the Tablet App}

The app developed for this project is a hybrid app, in contrast to a web app or a native app. A web app is written in HTML/CSS/JavaScript, languages that are ubiquitous. While easy for many people to code, web apps cannot access native mobile capabilities such as audio recording. A native app is written in the language of the device (ex: Objective C / Java). Although it can access all functionality of the mobile device, it works only on the specific platform (ex: IOS or android), so it takes more developer time and expertise to create an app for all users. A hybrid app is a trade-off between native and web app. It is written in the same languages as web apps are, permitting many people to leverage their web-building skills to produce a mobile app that may be run on different operating systems. A hybrid app is run within a WebView browser instance inside of a mobile device. Native functionality, such as camera or audio recording, is provided by plugins for the hybrid app. A hybrid app takes far less developer time and expertise to produce apps that are cross-platform.

Our app is an Ionic-Cordova hybrid app, which has a native look and feel. The Apache Cordova [18] platform is an open source framework for hybrid mobile app development that provides communication with native devices (ex: camera). The app programmer can utilize these devices through simple calls provided by plugins. In this open source environment, plugins, written in Objective $\mathrm{C}$ or Java, are provided by the community. Our app utilizes native functionality such as timer, camera, recorder, date-picker, geolocation and file reading/writing to capture data. The app produces .jpg files when photos are taken and .m4a files when recording are made. It is the Cordova platform that permits our app to control native functionality, permitting features such as auto update to modify an assessment when the question text file on which it is based is updated.
The Ionic framework [19], a powerful HTML5 SDK, sits on top of Cordova, supplying front end components in HTML/CSS/JavaScript that provide near-native look and feel for the app at a minimal cost of developer time. Ionic is built on AngularJS and utilizes design patterns such as factory [20], so that apps may be well-structured. Its popularity is due to the excellent UIs (user interfaces) it produces and the control over layout afforded by its Ionic grid.

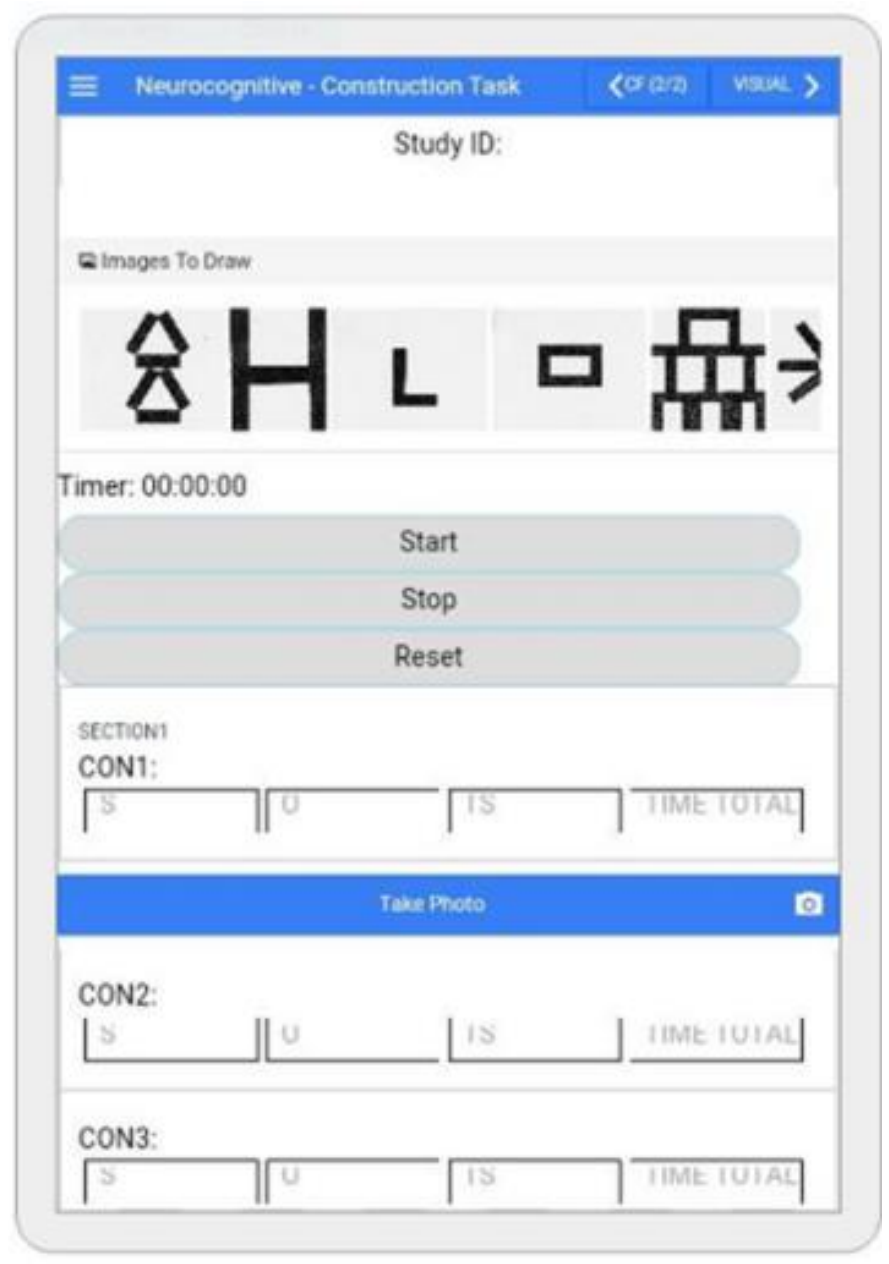

Fig. 1. A screen from the child assessment tablet app

\section{HOW THE APP WILL BE USED}

This app will be used in various studies with the Neuroassessment unit of KEMRI/Wellcome Trust Research Programme to look at the impact of ill-health on child development. Of specific interest would be to investigate the impact of neonatal insults and document their long-term impact. In the immediate term we intend to carry out a series of studies to evaluate the feasibility, acceptability and validity of the digital version of the Kilifi assessment scales. To begin this process, potential users were asked to offer their feedback on the use of this tablet format of assessment in comparison to the paper and pencil method. Three trained assessors 
administered scales to three participants aged between 3-5 years.

The assessors indicated an overall positive feedback in using the tablet compared to the paper and pencil version. Specifically, they reported that the tablet format was user friendly with clear instructions. Moreover, it was described as efficient, especially in reducing the administration time and minimizing scoring errors. They were particularly happy with the fact that spending less time writing (for instance when they had to note down the child's response) contributed to better engagement with the child. The small sample size precludes any direct comparison of scores but it seemed like there was more accuracy in standardizing timed tasks. However, the administrators also reported some concerns when using the tablet, including the need for extra vigilance when pressing the start button to make sure it had started. Additionally, one had to press "set time" before they could press the start timing button. Some of the issues raised may require modification of the tablet app or more training for the research assistants in using the tablet measures. These notwithstanding, the feedback given indicated that the tablet version might be easier to use, more acceptable and more efficient.

\section{Sample Question Text File}

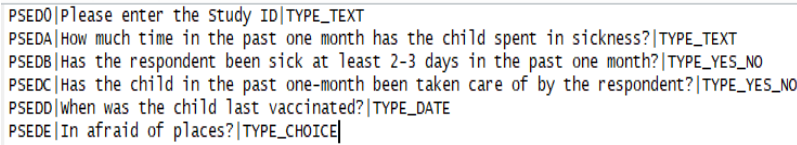

\section{Sample View of App}

\section{Based on Question File}

\begin{tabular}{|l|l|l|}
\hline PSED0 & Please enter the Study ID & Enter value \\
\hline PSEDA & $\begin{array}{l}\text { How much time in the past one month has the child } \\
\text { spent in sickness? }\end{array}$ & Enter value \\
\hline PSEDB & $\begin{array}{l}\text { Has the respondent been sick at least 2-3 days in the } \\
\text { past one month? }\end{array}$ & \\
\hline PSEDC & $\begin{array}{l}\text { Has the child in the past one month been taken care of } \\
\text { by the respondent? }\end{array}$ & \\
\hline PSEDD & When was the child last vaccinated? & Aug/11/2017 \\
\hline PSEDE & Is afraid of places? & 0 \\
\hline Save Answers
\end{tabular}

Fig. 2. Sample question text file and the screen that is rendered from that file

Following this process, we will work towards looking for validity and norming of these scales. The study would involve at least 600 children from within the Kilifi Demographic surveillance system. They will be stratified by age and sex. We will obtain interobserver and inter-rater reliability. Specific areas we would want to investigate would include looking at the levels of agreement between manually collected data and digitally recorded data. For instance, we will look at the differences in recorded times when we collect data manually as compared with when the data is captured by the app. This will allow for the evaluation of the incremental value of the app.

\section{FUTURE WORK}

This data collection app is being adapted for general use so that other researchers may write plain text files with survey questions, which will be automatically rendered into a similar data collection app tailored for their own use. The generic data collection app will be open-source and freely available to the public.

\section{CONCLUSION}

A tablet app was designed and developed by a team of cognitive researchers and health informaticists to replace paper and pencil early child cognitive assessments. During the entire design and development process, the users/researchers collaborated from Kenya while coders worked from the US. Current, freely available technology permitted the team to operate intercontinentally to produce a table app that was conformed to the Kenyan construct of intelligence. In this way researchers were able to modernize their cognitive assessment instruments while retaining their own local tests. Studies by experienced workers will compare paper and tablet versions of the Kilifi assessment scales to evaluate the impact of tablet staging. The researchers have the ability to modify the questions displayed on the app by merely modifying the text file from which the questions are drawn. The code for a generic form of this app will be available online in a public GitHub repository [21].

\section{REFERENCES}

[1] Walker, S. P., Wachs, T. D., Gardner, J. M., Lozoff, B., Wasserman, G. A., Pollitt, E., Carter, J. A. \& International Child Development Steering Group. Child development: risk factors for adverse outcomes in developing countries. Lancet, 369(9556), 2007, pp. 145-157.

[2] Grantham-McGregor, S., Cheung, Y. B., Cueto, S., Glewwe, P., Richter, L., Strupp, B., \& the International Child Development Steering Group. Developmental potential in the first 5 years for children in developing countries. Lancet, 369(9555), 2007, pp. 60-70.

[3] Abubakar, A., Van De Vijver, F. J., Mithwani, S., Obiero, E., Lewa, N., Kenga, S., ... \& Holding, P. (2007). Assessing developmental outcomes in children from Kilifi, Kenya, following prophylaxis for seizures in cerebral malaria. Journal of health psychology, 12(3), 417-430.

[4] Abubakar, A., Holding, P., Van de Vijver, F., Bomu, G., \& Van Baar, A. (2010). Developmental monitoring using caregiver reports in a resource - limited setting: the case of Kilifi, Kenya. Acta Paediatrica, 99(2), 291297.

[5] Pitchford, N. J., \& Outhwaite, L. A. Can touch screen tablets be used to assess cognitive and motor skills in early years primary school children? a cross-cultural study. Frontiers in psychology, 2016.

[6] Brombacher A. (2010). Malawi Early Grade Mathematics Assessment (EGMA): National Baseline Report. Malawi: USAID/Malawi and the Ministry of Education, Science and Technology.

[7] Gove A., Wetterberg A. (2011). The early grade reading assessment: an introduction, in The Early Grade Reading Assessment: Applications and Interventions to Improve Basic Literacy, eds Gove A., Wetterberg A., editors. (North Carolina: RTI Press; ), 1-38.

[8] Fernandes, M., Stein, A., Newton, C. R., Cheikh-Ismail, L., Kihara, M., Wulff, K., ... \& Ibanez, D. (2014). The INTERGROWTH-21st project neurodevelopment package: a novel method for the multi-dimensional 
assessment of neurodevelopment in pre-school age children. PLoS One, 9(11), e113360.

[9] Gladstone M, Lancaster GA, Umar E, Nyirenda M, Kayira E, van den Broek NR, Smyth RL The Malawi Developmental Assessment Tool (MDAT): the creation, validation, and reliability of a tool to assess child development in rural African settings. PLoS Med., 2010.

[10] Grigorenko E. L., Wenzel Geissler P., Prince R., Okatcha F., Nokes C., Kenny D. A., et al. The organisation of Luo conceptions of intelligence: a study of implicit theories in a Kenyan village. Int. J. Behav. Dev. 25, 2001, pp. 367-378.

[11] Prado EL, Hartini S, Rahmawati A, Ismayani E, Hidayati A, Hikmah N, Muadz H, Apriatni MS, Ullman MT, Shankar AH, Alcock KJ. Test selection, adaptation, and evaluation: a systematic approach to assess nutritional influences on child development in developing countries. $\mathrm{Br}$ J Educ Psychol., 2010, pp. 31-53.

[12] Kitsao-Wekulo PK, Holding PA, Taylor HG, Abubakar A, Connolly K. Neuropsychological testing in a rural African school-age population: evaluating contributions to variability in test performance.

[13] Sabanathan, S., Wills, B., \& Gladstone, M. Child development assessment tools in low-income and middle-income countries: how can we use them more appropriately? Archives of Disease in Childhood, 100(5), 2015, pp.482-488.
[14] Abubakar, A., Holding, P., Van Baar, A., Newton, C. R. J. C., \& van de Vijver, F. J. (2008). Monitoring psychomotor development in a resource limited setting: an evaluation of the Kilifi Developmental Inventory. Annals of tropical paediatrics, 28(3), 217-226.

[15] Alcock, K. J., Holding, P. A., Mung'ala-Odera, V., \& Newton, C. R. J. C. (2008). Constructing tests of cognitive abilities for schooled and unschooled children. Journal of Cross-Cultural Psychology, 39(5), 529551

[16] "An Introduction to Design Thinking PROCESS GUIDE", Hasso Plattner Institute of Design at Stanford, ModeGuideBootCamp2010.pdf, https://dschool-

old.stanford.edu/sandbox/groups/designresources/wiki/36873/attachmen ts/74b3d/ModeGuideBOOTCAMP2010L.pdf?sessionID=573efa71aea5 0503341224491c862e32f5edc0a9

[17] InVision app: https://www.invisionapp.com/

[18] Cordova platform: https://cordova.apache.org/

[19] Ionic framework: http://ionicframework.com

[20] Vlissides, J., Helm, R., Johnson, R., \& Gamma, E. (1995). Design patterns: Elements of reusable object-oriented software. Reading: Addison-Wesley, 49(120), 11.

[21] Github repostitories: https://github.com/ 